\title{
Numerical analysis of laser heating for laser assisted micro milling application
}

\begin{abstract}
The promising processing techniques of micro scale parts are very important in products miniaturization and functions enhancement. Combination of two or more processing techniques gives better processing performance especially when dealing with difficult-to-cut materials. For that reason, the combination of laser beam and micro milling process has been widely studied and proven efficient in reducing cutting force and tool life extension. However, this process needs a precise temperature control in order to eliminate heat effect generated by laser beam irradiation. In this study, temperature distributions are determined numerically to characterize the melted zone and heat affected zone geometry. From the results, the estimation of tool and micro milling cutter distance together with the allowable depth of cut are determined.
\end{abstract}

Keyword: Finite element analysis; Heat affected zone; LAMM; Pulsed laser; Titanium alloys 